\title{
Management of Septic Patients without the Consultation Services of Infectious Disease Specialists
}

Key words: sepsis, SIRS, septicemia, infectious disease specialists, nosocomial infection

Despite many advances in the antimicrobial therapy of various infectious diseases, sepsis remains a significant cause of morbidity and mortality in hospitalized patients (1-3). The usual clinical course of sepsis progresses from unrecognized infection through a period of clinically apparent infection with signs and symptoms of systemic inflammatory responses to a lifethreatening condition of septic shock and multiple organ failure (4). According to our recent observation in patients with systemic inflammatory response syndrome (SIRS), there were stepwise increases in mortality rates in the progression from sepsis to severe sepsis, and finally septic shock; the mortality rates being 30.6 per cent, 53.3 per cent, and 64.0 per cent, respectively (1). For many years it has been known that early recognition of the systemic inflammatory signs associated with infection and prompt detection of the blood-borne pathogens are mandatory for reducing the mortality of sepsis. In a study on surgical sepsis, Wilson demonstrated that if sepsis was not diagnosed and operated upon aggressively prior to the development of shock or organ failure, the mortality rate rose from 12 per cent to $64-88$ per cent (5). In addition, a delayed antibiotic therapy would increase the mortality of sepsis (6).

In this issue of Internal Medicine, Dr. Aoki and colleagues (7) present the epidemiology of sepsis patients and practice of their management in a tertiary care teaching hospital in Japan.

See also p 901.

In a prospective observational study, they have reviewed 73 patients who had a positive blood culture and who met the diagnosis criteria of sepsis proposed by the Consensus Conference of the American College of Chest Physicians and Society of Critical Care Medicine (8). By using this definitive enrollment criteria in this prospective study, the authors could select rather a homogenous population of "bacteremic sepsis" than a population of simple "sepsis" that includes a broad spectrum and different severity of patients with infectious diseases $(8$, 9). The "bacteremic sepsis" in this study could be called "septicemia", but this medical term is becoming obsolete after the Consensus Conference recommended that the term not be used (8).

It must be pointed out that the patients enrolled in this study
(7) were treated in non-ICU wards of different clinical services by physicians who were not specialized in infectious diseases. As in most Japanese teaching hospitals where an independent subspecialty of infectious diseases does not exist, their hospital had no resource of infectious disease consultation services.

In this study, the overall mortality rate was 34 per cent. Fiftysix patients (77\%) had hospital-acquired sepsis that developed while the patient was hospitalized for medical problems other than sepsis. Methicillin-resistant Staphylococcus aureus (MRSA) was isolated by blood culture in eleven patients of which six patients (55\%) died. Twenty-three patients (32\%) had septic shock, and it is not surprising that the mortality rate of patients with septic shock was 52 per cent that was much higher than that of non-shock patients (26\%).

Although the patients were treated without a consultation service of infectious disease specialists and intensive care physicians, the overall outcome of the sepsis patients in this study was in an acceptable range as compared with the mortality rates shown in other sepsis studies (1-3). However, if these patients had been managed with an involvement of these specialists, the development of hospital-acquired sepsis and septic shock could have been reduced and the outcome of the bacteremic sepsis could have been better. As pointed out in this paper, it seems that the physicians (or surgeons) did not pay too much attention to their patients when clinically defined sepsis developed. Out of four vital signs (pulse rate, blood pressure, body temperature, and respiratory rate) of the sepsis patients, the physicians recorded in a chart only 1.67 vital signs on average. The first blood culture sampling was delayed until two to three days after the onset of sepsis, and the mean number of blood cultures taken was 1.5 in the entire course of sepsis. Only 21 patients $(29 \%)$ had a multiple set blood cultures.

The reasons for this rather unsatisfactory practice for an early diagnosis of bacteremic sepsis are obvious. The doctor who has to take care of sepsis patients in that hospital could be a hematologist, a neurologist, a cardiologist, a general surgeon, or even a cardiac surgeon, who has limited knowledge and experience in the management of sepsis. Furthermore, routine consultation with an infectious disease specialist was not available. I regret to admit that this is rather a common practice in many of the Japanese teaching hospitals including our hospital in Tokyo.

In 1995 the Japanese Association for Infectious Diseases established a program to certify a trained member of the association to be a fellow. Currently there are 757 fellows of the 
association whose primary specialty is either internal medicine or pediatrics. In addition, a committee sponsored by 12 infection-related academic societies in Japan is recognizing the position of "an infection control doctor (ICD)", and 1,121 doctors have been certified to date. The introduction of these "infectious disease specialists", however, has basically not changed the clinical practice of infection management to date, as few specialists are working full-time for infectious disease consultation. Few infection fellow members or ICDs are asked for his/her recommendation for the diagnosis and treatment of infectious diseases by other doctors. In most institutions in Japan, when a nosocomial surgical infection develops in a postoperative patient, it is customary for the surgeon who operated on that particular patient to decide the antimicrobial therapy and no other professional would make comments on the surgeon's decision. However, we know well that modern management of sepsis patients requires an involvement of many specialists (10).

In an era of growing resistant pathogens and increasing nosocomial infections, it is high time change our mind and introduce a truly workable system of infectious diseases subspecialty and consultation services into Japanese practice of medicine.

Physician, heal thyself. Bible; Luke 4 :23

Naoki AIKAWA, MD, FACS

The Department of Emergency and Critical Care Medicine School of Medicine, Keio University 35 Shinanomachi, Shinjuku-ku, Tokyo 160-8582

\section{References}

1) Sun D, Aikawa N. The natural history of the systemic inflammatory response syndrome and the evaluation of SIRS criteria as a predictor of severity in patients hospitalized through emergency services. Keio J Med 48: 28-37, 1999

2) Brun-Buisson C, Doyon F, Darlet J, et al. Incidence, risk factors, and outcome of severe sepsis and septic shock in adults. JAMA 274: 968974, 1995.

3) Rangel-Frausto MS, Pittet D, Costigan M, Hwang T, Davis CS, Wenzel RP. The natural history of the systemic inflammatory response syndrome (SIRS): A prospective study. JAMA 273: 117-123, 1995.

4) Munford RS. Sepsis and Septic Shock. in: Harrison's Principles of Internal Medicine. 14 th ed. Fauchi AS, Braunwald E, Isselbacher KJ, et al. Eds. McGraw-Hill, New York, 1998: 776-780.

5) Wilson RF. Etiology, diagnosis, and prognosis of positive blood cultures. Am Surg 47: 112-115, 1981.

6) Pittet D, Thievent B, Wenzel RP, Li N, Auckenthaler R, Suter PM. Bedside prediction of mortality from bacteremic sepsis: A dynamic analysis of ICU patients. Am J Respir Crit Care Med 153: 684-693, 1996.

7) Aoki Y, Fukuoka M, Kusaba K, et al. Current practice of management of bacteremic sepsis: A study in a tertiary care teaching hospital in Japan. Intern Med 39: 901-909, 2000.

8) Bone RC, Balk RA, Cerra FB, et al. The American College of Chest Physicians/Society of Critical Care Medicine Consensus Conference Committee: Definitions for sepsis and organ failure and guidelines for the use of innovative therapies in sepsis. Chest 101: 1644-1655, 1992.

9) Bone RC. Sepsis, sepsis syndrome, and the systemic inflammatory response syndrome (SIRS). Gulliver in Laputa [editorial; comment]. JAMA 273: 155-156, 1995.

10) Wheeler AP, Bernard GR. Treating patients with severe sepsis. New Engl J Med 340: 207-214, 1999. 